\title{
Differential expression of natural antisense transcripts during liver development in embryonic mice
}

\author{
MITSURU CHIBA \\ Department of Biomedical Sciences, Division of Medical Life Sciences, Hirosaki University \\ Graduate School of Health Sciences, Hirosaki, Aomori 036-8564, Japan
}

Received August 15, 2014; Accepted September 8, 2014

DOI: $10.3892 / \mathrm{br} .2014 .363$

\begin{abstract}
The mature adult liver is a metabolizing and synthesizing organ consisting of hepatic lobules. The liver development during fetal stages is induced by the interaction between hepatoblast cells derived from the endoderm and stellate cells and/or endothelial cells derived from the mesoderm. Recently, the association of non-coding RNAs, such as microRNAs, in the liver development process has been reported. In the present study, the gene expression changes were investigated of natural antisense transcripts (NATs), a type of non-coding RNA, during the liver development using livers from embryonic day (E) 14, E17, E19 and newborn (NB). The upregulated NATs of 87 genes and the downregulated NATs of 26 genes were identified when the expression was $>5.0$-fold up/downregulated NATs at E17, E19 or NB compared to those at E14, as examined by microarray analysis. Among these NATs, strand-specific reverse transcription-quantitative polymerase chain reaction analyses validated that the NAT expression of Fga, Fgb, Fgg, F2, Apoal, Fabpl, C3 and Proc, which have metabolic and synthesizing functions in the adult liver, increased, whereas the NAT expression of Ermap and Tfrc, which function in the hematopoiesis, decreased during the developmental stages. These results indicate that the differential expression of several NATs is induced during the process of liver development, suggesting that these NATs may be involved in the regulation of gene expression and functional conversion from a hematopoietic to a metabolic organ in the developmental stages of the liver.
\end{abstract}

Correspondence to: Dr Mitsuru Chiba, Department of Biomedical Sciences, Division of Medical Life Sciences, Hirosaki University Graduate School of Health Sciences, 66-1 Hon-cho, Hirosaki, Aomori 036-8564, Japan

E-mail: mchiba32@cc.hirosaki-u.ac.jp

Key words: liver development, natural antisense transcripts, non-coding RNA

\section{Introduction}

The liver is an organ consisting of hepatocytes, stellate cells, endothelial cells and Kupffer cells. It consists of hepatic lobules as basic units and plays important roles in the production of bile acids, proteosynthesis, detoxification, glycogen storage and hematopoiesis. Injured or partially hepatectomized livers can recover their size and function by enlarging hepatocytes (1).

During the process of liver development, hepatoblast cells differentiate from the endoderm and stellate cells, and endothelial cells differentiate from the mesoderm. The construction of the liver in the fetus is induced by the interaction among cells through hormones and/or cytokines. Once a fertilized egg implants in the uterus, the ectoderm, endoderm and mesoderm differentiate from the inner clump of cells. The endoderm forms the foregut under the control of the forkhead box A2 transcription factor and GATA binding protein 4, and a section of the foregut forms hepatoblast cells $(2,3)$. Fibroblast growth factor secreted from the transverse septum and bone morphogenetic protein secreted from the heart endoderm stimulate endoderm cells of the foregut to differentiate into hepatoblast cells $(4,5)$. Hepatocyte growth factor secreted from endothelial cells enhances the proliferation of hepatoblast cells and the process of liver development $(6,7)$.

Mature hepatocytes and bile duct epithelial cells are derived together from hepatoblast cells. The change in the gene expression of CCAAT enhancer binding protein $\alpha$, a liver-specific transcription factor, affects the differentiation into mature hepatocytes and bile duct epithelial cells (8). In addition, Nodal, which belongs to the transforming growth factor- $\beta$ (TGF- $\beta$ ) superfamily, is involved in the selection of mature hepatocytes or bile duct epithelial cells for the differentiation (9). In general, TGF- $\beta$ suppresses the cell proliferation. The cells destined to be bile duct epithelial cells stop proliferating due to TGF- $\beta$ stimulation and form bile duct cells. By contrast, the cells destined to be hepatocytes continue to proliferate by avoiding TGF- $\beta$ stimulation. Functional non-coding RNAs, such as microRNAs (miRs), have been reported to be involved in the regulation of the TGF- $\beta$ pathway. Rogler et al (10) reported that $m i R-23 b$ suppressed the expression of Smad, which functions downstream of the TGF- $\beta$ pathway and inactivates the TGF- $\beta$ pathway. In addition, Hand et al (11) reported that $m i R-30$ participated in the formation of bile ducts from hepatoblast cells in mice. 
Therefore, it is important to investigate the gene expression of non-coding RNAs during the development of the fetal liver to understand the mechanisms of the liver development.

Natural antisense transcripts (NATs), which are transcribed from the DNA strand opposite to the sense strand, are involved in controlling the gene expression in eukaryotes (12). NATs are usually non-coding RNAs. The study by Matsui et al (13) reported that NATs participated in maintaining the stability of the inducible nitric oxide synthase mRNA in the liver. In addition, our previous study (14) reported that the expression of NATs was up/downregulated in the regenerating livers of mice. However, the effects of NAT expression during the liver development remain unclear.

In the present study, the expression profiles of NATs were investigated using samples of the developing liver from mice. The aim of the study was to identify the up/downregulated NATs during liver development.

\section{Materials and methods}

Liver samples. Livers at different developmental stages, i.e., embryonic day (E) 14, E17, E19 and newborn (NB), were obtained from the C57BL/6 mice at the RIKEN BioResource Center (Tsukuba, Ibaraki, Japan). All the animal experiments were carried out according to the RIKEN Guidelines for the care and use of experimental animals. For the RNA analysis, livers at different developmental stages (each $n=3$ ) were collected, frozen immediately in liquid nitrogen and stored at $-80^{\circ} \mathrm{C}$ until use.

Total RNA extraction and quality check. Total RNAs from frozen livers were isolated using Isogen (Nippon Gene, Tokyo, Japan), according to the manufacturer's instructions. The quality and concentration of total RNAs were assessed using the NanoDrop spectrophotometer (NanoDrop Technologies, Wilmington, DE, USA), according to manufacturer's instructions. All the total RNA samples had 260/280 nm absorbance ratios of 1.8-2.0. The Agilent 2100 Bioanalyzer (Agilent Technologies, Santa Clara, CA, USA) and the RNA 6000 Nano LabChip kit (Agilent Technologies) were used to evaluate the RNA integrity. The total RNAs examined were shown as RNA integrity numbers $>8.1$. On the basis of the Bioanalyzer's instructions, total RNAs obtained were judged to be suitable for microarray analysis.

Microarray analysis. Agilent 44K x 4 mouse sense and antisense custom-microarray slides [Agilent eArray Design ID, 021137; produced by Tsukuba GeneTechnology Laboratories (http://www.tsukuba-genetech.com), Ibaraki, Japan] and Cy3-labeled cDNA were used. The microarray analysis was performed as described previously (14).

The expression profiles at each stage (E17, E19 and NB) were compared against those at E14 using GeneSpring GX12 software (Agilent Technologies). The Kruskal-Wallis test was performed to identify the genes with significant differences at each time point; $\mathrm{P}<0.05$ was considered to indicate a statistically significant difference. The upregulated and downregulated NATs were selected with fold changes $(>5.0)$ at each time point against E14 and with signal intensities $>100.0$.
Table I. Identification of up- or downregulated natural antisense transcripts during liver development by microarray analyses.

\begin{tabular}{ll}
$\begin{array}{l}\text { Gene } \\
\text { regulation }\end{array}$ & \multicolumn{1}{c}{ Gene symbol } \\
\hline Up & Syt10, Fgg, Serpinala, Rbp4, Acaa2, Ttll10, Acadm, \\
(87 genes) & Scp2, Igfbp2, Elovl5, Angptl3,Serpina1c, Cyp2c67, \\
& Arhgef10l, Tmprss9,Itih3, Cpb2, Ahsg, H2-Q1, Ly6e, \\
& Crot, Csad, Fga, Cyp2c40, Lyz1, Cfb, Abhd2, \\
& Serpina1b, F2, Olfr91, Serpinald, Acat1, H2-T23, \\
& Selenbp2, Itsn1, Camp, Phyh, Zfp62, Lamp2, Serpinc1, \\
& Elovl2, Mpc1, Apoe, Pygl, F10, Pate4, LOC641235, \\
& Fgb, Spata21, Vtn, Lgals9, Fads2, B2 m, Trf, Fabp1, \\
& Hdc, Il18, P4hb, Proc, Pm20d2, Fbxw5, Apof, C3, Fah, \\
& Pzp, Ech1, Bdh1, Kng1, Sepp1, Apoa2, Gcat, H2-T3, \\
& Sdr42e1, Nudt7, Apoh, Pah, Crp, Apoa1, Kcnab3, Ctsl, \\
& Adh1, Mypop, Lpl, Alb, Fgfbp1, S100a9 and Acad11 \\
& Slc25a37, Rpl4, Pklr, Cbln2, Ccl5, Car2, Pa2g4, Cpox, \\
& Ermap, Ptcd1, Hemgn, Ncl, Was, Scn4b, Gpr150, Sypl, \\
& Klf1, Prdx2, Nop56, Tfrc, Cdk13, Agap3, Asns, Yod1, \\
& Rhag and Magoh
\end{tabular}

Strand-specific reverse transcription quantitative polymerase chain reaction $(R T-q P C R)$. Strand-specific RT-qPCR was performed using total RNA obtained from livers at different developmental stages to confirm the expression patterns of NATs during the liver development.

First-strand cDNAs derived from NATs or mRNA were synthesized using a forward or a reverse primer, $18 \mathrm{~S}$ ribosomal RNA (rRNA) reverse primer and Reverse Transcriptase (Promega, Madison, WI, USA), according to the manufacturer's instructions. The mixtures were incubated at $50^{\circ} \mathrm{C}$ for $60 \mathrm{~min}$. The resulting cDNA was incubated with RNase A at $99^{\circ} \mathrm{C}$ for $5 \mathrm{~min}$ and at $37^{\circ} \mathrm{C}$ for $60 \mathrm{~min}$ to digest the RNA.

qPCR of NATs or mRNA was performed using first-strand cDNAs, FastStart Universal SYBR-Green Master (Roche Diagnostics, Basel, Switzerland) and primer pairs. qPCR analyses were performed using the StepOne Plus Real-Time PCR system (Life Technologies, Carlsbad, CA, USA) for $1 \mathrm{~min}$ at $95^{\circ} \mathrm{C}$, followed by 40 cycles each of $95^{\circ} \mathrm{C}$ for $15 \mathrm{sec}$ and $60^{\circ} \mathrm{C}$ for $60 \mathrm{sec}$, following the manufacturer's instructions. $\mathrm{Ct}$ values were averaged and normalized to that of $18 \mathrm{~S}$ rRNA. The relative expression ratio was determined by the $\Delta \Delta \mathrm{Ct}$ comparative threshold method. The results of qPCR are presented as the mean \pm standard error of samples.

\section{Results and Discussion}

Previously, several non-coding RNAs have been reported to be involved in the liver development (15). In the present study, the expression of NATs was investigated to obtain further information on the mechanisms occurring during the liver development. The up/downregulated NATs during the liver development were identified using mouse sense/antisense microarray slides.

The upregulated NATs of 87 genes were identified with $>5.0$-fold changes in E17, E19 or NB compared to those at E14. In addition, the downregulated NATs of 26 genes were identified (Table I). These results indicate that the expression 

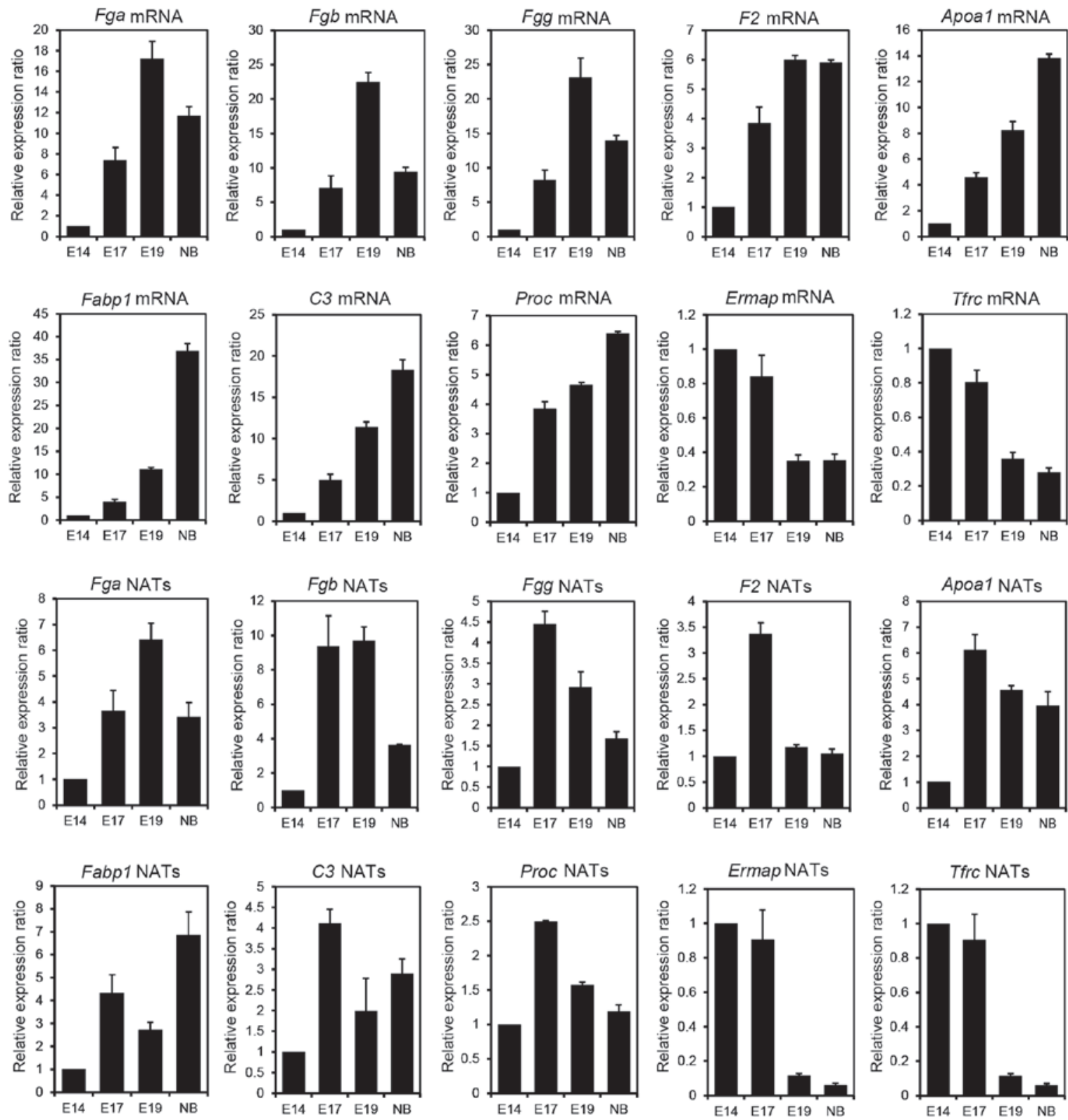

Figure 1. A comparison of natural antisense transcript (NAT) and mRNA expression at various liver developmental stages by strand-specific reverse transcription-quantitative polymerase chain reaction (RT-qPCR). First-strand cDNAs derived from NATs or mRNA were synthesized using a forward primer and a reverse primer (Table II). Strand-specific RT-qPCR was performed to investigate the values of the expressed NATs. The Ct values obtained were averaged and normalized to that of $18 \mathrm{~S}$ ribosomal RNA. The relative expression ratio was determined by the $\Delta \Delta \mathrm{Ct}$ comparative threshold method and calculated using the values at embryonic day (E) 14 as 1.0. qPCR results are presented as the mean \pm standard error of samples (each n=3). NB, newborn; Fga, fibrinogen $\alpha$ chain; $F g b$, fibrinogen $\beta$ chain; Fgg, fibrinogen $\gamma$ chain; F2, coagulation factor II; Apoal, apolipoprotein A-I; Fabpl, fatty acid binding protein 1; C3, complement component 3; Proc, protein C; Ermap, erythroblast membrane-associated protein; Tfrc, transferrin receptor.

of several NATs changed, suggesting important roles during the liver development.

Several NATs described above were validated by the strand-specific RT-qPCR to verify the expression of NATs obtained by microarray analyses. The expression of fibrinogen $\alpha$ chain $(F g a)$, fibrinogen $\beta$ chain $(F g b)$, fibrinogen $\gamma$ chain $(F g g)$, coagulation factor II $(F 2)$, fatty acid binding protein 1 (Fabpl), complement component 3 (C3), apolipoprotein A-I (Apoal) and protein C (Proc) NATs was examined and these were observed as upregulated candidate NATs in the progression of the liver development. As shown in Fig. 1, the expression of $F g a, F g b$ and $F g g$, known as the fibrinogen family, increased in E17 and/or E19 compared to that in E14. This result indicates that these NATs may play important roles in E17 and/or E19 stages. F2, known as thrombin, is a blood coagulation factor [Online Mendelian Inheritance in Man (OMIM): 176930] and Proc inhibits the blood coagulation (OMIM: 612283). The expression of F2 and Proc NATs increased 3.4-fold and 2.5 -fold, respectively, in E17 compared to that in E14 and subsequently decreased. This result indicates that $F 2$ and Proc NATs may function only during the E17 stage. Fabpl is involved in the transport and metabolism of fatty acids by binding to free fatty acids (OMIM: 134650). C3 is a protein that mediates immunoreactions and acts during the bacteriolysis by binding to the bacterial surface (OMIM: 120700). The increased levels of Fabpl and C3 NATs had two phases: 
Table II. Primer sequences for the detection of natural antisense transcripts.

\begin{tabular}{|c|c|c|c|}
\hline Primer name & Sequences & Size (mer) & PCR products $(\mathrm{bp})$ \\
\hline Apoal & & & 60 \\
\hline Forward & 5'-GAAGGATTTCGCTAATGTGTATGTG-3' & 25 & \\
\hline Reverse & 5'-GACACATAGTCTCTGCCGCT-3' & 20 & \\
\hline$C 3$ & & & 60 \\
\hline Forward & 5'-CACAGAATCTATGGTGGTTTATGG-3' & 24 & \\
\hline Reverse & 5'-TTTATTAGAGGGCTGGGCTGTA-3' & 22 & \\
\hline$F 2$ & & & 60 \\
\hline Forward & 5'-AAATACGGCTTCTACACGCAT-3' & 21 & \\
\hline Reverse & 5'-ATCAATGACTTTCTGTATCCACC-3' & 23 & \\
\hline Fabpl & & & 60 \\
\hline Forward & 5'-GTGACTGAACTCAATGGAGACAC-3' & 23 & \\
\hline Reverse & 5'-GTAGACAATGTCGCCCAATGTCA-3' & 23 & \\
\hline Fga & & & 60 \\
\hline Forward & 5'-CAGAGCAATGTCCGGGCT-3' & 18 & \\
\hline Reverse & 5'-GATATCAATATCCACCTCCAGGC-3' & 23 & \\
\hline$F g b$ & & & 60 \\
\hline Forward & 5'-TTCTCATTGAAATGGAGGACTGGA-3' & 24 & \\
\hline Reverse & 5'-TGAAGCCTCCATAATGTGCCT-3' & 21 & \\
\hline Fgg & & & 60 \\
\hline Forward & 5'-GATTTTGAGTAAGTACCACCTTGG-3' & 24 & \\
\hline Reverse & 5'-GATGAACAAATGTCACGCAGG-3' & 21 & \\
\hline Proc & & & 60 \\
\hline Forward & 5'-AAACGAGACACAGACTTAGAAGATG-3' & 25 & \\
\hline Reverse & 5'-CGTTCCGTTGACTATCCTTG-3' & 20 & \\
\hline Ermap & & & 60 \\
\hline Forward & 5'-CTGCTACAGGCTCTTTCCAA-3' & 20 & \\
\hline Reverse & 5'-GTTGGTTCTCTTTCTCTAGGG-3' & 21 & \\
\hline Tfrc & & & 60 \\
\hline Forward & 5'-TGCTACTCAGTCCCTTCAG-3' & 19 & \\
\hline Reverse & 5'-ACGGTGATACTCATACTGGTAC-3' & 22 & \\
\hline $18 S$ rRNA & & & 85 \\
\hline Forward & 5'-CACGGACAGGATTGACAGATTG-3' & 22 & \\
\hline Reverse & 5'-CAAATCGCTCCACCAACTAAGA-3' & 22 & \\
\hline
\end{tabular}

bp, base pair; $C 3$, complement component $3 ; F 2$, coagulation factor II; Fabpl, fatty acid binding protein $1 ; F g a$, fibrinogen $\alpha$ chain; $F g b$, fibrinogen $\beta$ chain; $F g g$, fibrinogen $\gamma$ chain; Proc, protein C; Ermap, erythroblast membrane-associated protein; Tfrc, transferrin receptor; $18 S$ rRNA, 18s ribosomal RNA.

One peak was observed at E17 and another was observed at NB, suggesting that Fabpl and C3 NATs may be involved in the regulation of gene expression at E17 following birth and may indicate further high expression in the adult liver. The albumin $(A l b)$ and alcohol dehydrogenase 1 (Adh 1$)$ genes shown in Table I were involved in liver metabolism, indicating that these NATs may have important roles in the fetal and adult liver. In addition, the sense transcripts (mRNAs) of the genes described above were confirmed to be upregulated during liver development (Fig. 1). These findings indicate that these NATs and mRNAs are co-expressed in the developmental stages and may participate in the regulation of gene expression, such as the maintenance of the mRNA stability (13).

By contrast, strand-specific RT-qPCR was performed to confirm the expression of erythroblast membrane-associated protein (Ermap), transferrin receptor (Tfrc) p90 and CD71 as downregulated candidate NATs. Ermap functions as a cell-adhesion or a receptor molecule of erythroid cells (OMIM: 609017). Tfrc is an essential gene that imports iron into erythroid cells (OMIM: 190010). As shown in Fig. 1, the expression of Ermap and Tfrc NATs was increased up to the E17 stage but showed markedly decreased expression following the E19 stage. In addition, the NATs of hemogen (Hemgn), which regulate the proliferation and differentiation of hematopoietic cells (OMIM: 610715), were downregulated during liver development (Table I). These results indicate that decreased NATs of Ermap, Hemgn and Tfrc during liver development may be involved in the regulation of the hematopoietic function.

In our previous study (14), we showed that several NATs, including Fga, Fgb, Fgg, inter- $\alpha$-trypsin inhibitor heavy chain 3 (Itih3) and cathepsin L $(\mathrm{Ctsl})$, were upregulated when the NAT expression changes were examined during liver regeneration using $70 \%$ partial hepatectomized mice. Notably, Fga, Fgb, Fgg, Itih 3 and $C t s l$ NATs were upregulated during liver development 
in the present study (Table I). This result indicates that these NATs may act in the dramatic functional conversion of the liver, such as during the liver regeneration and development.

In conclusion, several NATs were revealed to be up/downregulated during liver development. Certain NATs were co-expressed with mRNA of the same genes. Therefore, NATs may be involved in the co-regulation with these mRNAs. The fetal liver functions mainly as a hematopoietic organ. During the developmental progress, the hematopoietic function declines and the function conversion is subsequently induced from a hematopoietic organ to a metabolic and synthesizing organ. NATs may be involved in this functional conversion of the fetal liver. Further studies are required to understand the mechanisms of these NATs during the liver development.

\section{Acknowledgements}

I am grateful to Ms. Noriko Hiraiwa (RIKEN BioResource Center) for offering the samples. The present study was supported in part by the Grants-in-Aid from the Ministry of Education, Culture, Sports, Science and Technology of Japan and the Hirosaki University Grant for Exploratory Research by Young Scientists.

\section{References}

1. Higgins GM and Anderson RM: Experimental pathology of the liver: restoration of the liver of the white rat following partial surgical removal. Arch Pathol 12: 186-202, 1931.

2. Bossard P and Zaret KS: GATA transcription factors as potentiators of gut endoderm differentiation. Development 125: 4909-4917, 1998.

3. Cirillo LA, Lin FR, Cuesta I, Friedman D, Jarnik M and Zaret KS: Opening of compacted chromatin by early developmental transcription factors HNF3 (FoxA) and GATA-4. Mol Cell 9: 279-289, 2002.
4. Jung J, Zheng M, Goldfarb $M$ and Zaret KS: Initiation of mammalian liver development from endoderm by fibroblast growth factors. Science 284: 1998-2003, 1999.

5. Rossi JM, Dunn NR, Hogan BL and Zaret KS: Distinct mesodermal signals, including BMPs from the septum transversum mesenchyme, are required in combination for hepatogenesis from the endoderm. Genes Dev 15: 1998-2009, 2001.

6. Schmidt C, Bladt F, Goedecke S, et al: Scatter factor/hepatocyte growth factor is essential for liver development. Nature 373: 699-702, 1995.

7. Ishikawa KS, Masui T, Ishikawa $\mathrm{K}$ and Shiojiri N: Immunolocalization of hepatocyte growth factor and its receptor (c-Met) during mouse liver development. Histochem Cell Biol 116: 453-462, 2001

8. Shiojiri N, Takeshita K, Yamasaki H and Iwata T: Suppression of C/EBP alpha expression in biliary cell differentiation from hepatoblasts during mouse liver development. J Hepatol 41: 790-798, 2004

9. Vincent SD, Dunn NR, Hayashi S, Norris DP and Robertson EJ: Cell fate decisions within the mouse organizer are governed by graded Nodal signals. Genes Dev 17: 1646-1662, 2003.

10. Rogler CE, Levoci L, Ader T, Massimi A, Tchaikovskaya T, Norel R and Rogler LE: MicroRNA-23b cluster microRNAs regulate transforming growth factor-beta/bone morphogenetic protein signaling and liver stem cell differentiation by targeting Smads. Hepatology 50: 575-584, 2009.

11. Hand NJ, Master ZR, Eauclaire SF, Weinblatt DE, Matthews RP and Friedman JR: The microRNA-30 family is required for vertebrate hepatobiliary development. Gastroenterology 136: 1081-1090, 2009.

12. Faghihi MA and Wahlestedt C: Regulatory roles of natural antisense transcripts. Nat Rev Mol Cell Biol 10: 637-643, 2009.

13. Matsui K, Nishizawa M, Ozaki T, et al: Natural antisense transcript stabilizes inducible nitric oxide synthase messenger RNA in rat hepatocytes. Hepatology 47: 686-697, 2008.

14. Chiba M, Yasue $\mathrm{H}$ and Ohkohchi N: Gene expression profiling of sense and antisense transcripts in liver regeneration by microarray analysis. Biomed Rep 1: 383-388, 2013.

15. Tzur G, Levy A, Meiri E, et al: MicroRNA expression patterns and function in endodermal differentiation of human embryonic stem cells. PLoS One 3: e3726, 2008. 